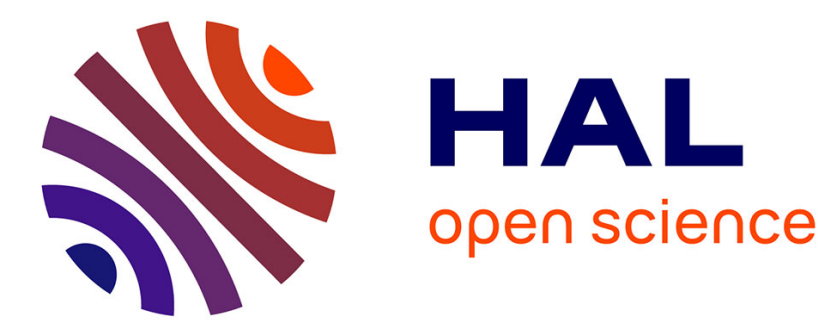

\title{
Coherently excited atoms in external electric fields
}

Maurice Lombardi, Marc Giroud, Joseph Macek

\section{To cite this version:}

Maurice Lombardi, Marc Giroud, Joseph Macek. Coherently excited atoms in external electric fields.

Physical Review A, 1975, 11, pp.1114-1117. 10.1103/PHYSREVA.11.1114 . hal-00974329

\section{HAL Id: hal-00974329 \\ https://hal.science/hal-00974329}

Submitted on 6 Apr 2014

HAL is a multi-disciplinary open access archive for the deposit and dissemination of scientific research documents, whether they are published or not. The documents may come from teaching and research institutions in France or abroad, or from public or private research centers.
L'archive ouverte pluridisciplinaire HAL, est destinée au dépôt et à la diffusion de documents scientifiques de niveau recherche, publiés ou non, émanant des établissements d'enseignement et de recherche français ou étrangers, des laboratoires publics ou privés. 


\title{
Coherently excited atoms in external electric fields*
}

\author{
Maurice Lombardi and Marc Giroud \\ Laboratoire Spectrometrie Physique, Université Scientifique et Mecicale de Grenoble, B. P. 53, 38041 Grenoble-Cedex, France \\ Joseph Macek $^{\dagger}$ \\ Fachbereich Physik der Freien Universität Berlin, 1 Berlin 33, Boltzmannstrasse 20, Germany \\ (Received 14 May 1974)
}

\begin{abstract}
Various manifestations of coherent mixtures of even- and odd-parity eigenstates of collision-excited atomic hydrogen in external electric fields are discussed on general grounds. We show that when evenand odd-parity states are coherently excited, application of an electric field perpendicular to the velocity axis induces orientation in the originally unoriented atoms. Circular polarization of the decay radiation is an observable consequence of the orientation.
\end{abstract}

Recently, $\mathrm{Eck}^{1}$ proposed a measurement of $\mathrm{Ly}-\alpha$ radiation designed to detect coherence of the $n=2$ $S$ and $P$ states of beam-foil-excited hydrogen. The proposed experiment ${ }^{2}$ consists of measuring the intensity of Ly- $\alpha$ emitted by atoms in an electric field alternately parallel and antiparallel to the incident-beam velocity $\vec{v}$. The difference of the two intensities is directly proportional to the magnitude of the density-matrix element $\sigma_{S P}$. In this Comment we explore some further manifestations of $S P$ coherence of hydrogenlike ions in electric fields. The main point we emphasize is that the emitted radiation is circularly polarized along the $\overrightarrow{\mathrm{V}} \times \overrightarrow{\mathrm{E}}$ axis if there is $S P$ coherence, even if $\overrightarrow{\mathrm{E}}$ is perpendicular to $\vec{v}$. This phenomenon, due to the first-order Stark effect, is somewhat similar to the production of atomic orientation by the secondorder Stark effect $t^{3}$ in nonhydrogenic atoms by an electric field neither parallel nor perpendicular to the beam axis. Since both effects coexist in hydrogenic ions, we shall discuss how effects due to $S P$ coherence are distinguished from those due to non-SP-coherent excitation.

Our discussion utilizes the concepts of alignment and orientation. Orientation of an atomic state is specified by the mean value of the electronic angular momentum $\vec{J}$ and alignment by the mean value of second-rank tensors constructed from the components of $\vec{J}$. The mean value of the tensor component $3 J_{z}^{2}-J^{2}$ is of particular interest here and will be referred to simply as the alignment.

In this study it is desirable to avoid the use of perturbation theory since a large coherence signal requires fields sufficiently strong that the $S$ and $P$ states are completely mixed. We will demonstrate the existence of the various coherence properties using only general symmetry arguments, and defer any results of detailed calculations and specific applications to subsequent publications. The mathematical arguments will be illustrated by a simple pictorial representation of the various effects.

The intensity of radiation is expressed in the for $\mathrm{m}^{4}$

$$
I(t)=C \sum_{i j}\left(i\left|\exp \left(i H^{\dagger} t \mid \hbar\right) S \exp (-i H t \mid \hbar)\right| j\right) \sigma_{j i},
$$

where $S$ is the monitoring operator, $H=H_{0}+V$ is the Hamiltonian, and $C$ is a constant. The unperturbed Hamiltonian includes the fine and hyperfine structure and a damping term. The damping term is a diagonal matrix whose elements are $-\frac{1}{2} \Gamma_{j j}$, where $\Gamma_{j j}$ is the decay width of the $j$ th state. The perturbation Hamiltonian $V$ is the electric potential energy $-e \overrightarrow{\mathrm{E}} \cdot \overrightarrow{\mathrm{r}}$. The measurement operator is given in terms of a real or complex polarization vector $\hat{\epsilon}$ by

$$
\left.S_{\hat{\epsilon}}=\sum_{0} \hat{\epsilon} \cdot \overrightarrow{\mathbf{r}}^{\prime} \mid 0\right)\left(0 \mid \hat{\epsilon}^{*} \cdot \overrightarrow{\mathbf{r}},\right.
$$

where 0 refers to the quantum numbers of the lower level of the observed optical transition and the summation is over all fine and hyperfine sublevels of this lower level. Since $S$ is quadratic in $\vec{r}$, it commutes with the operation $P$ of inversion of electronic coordinates. We will use this inversion symmetry to demonstrate that the difference between the intensity $I(\vec{E}, \vec{V})$ with $\vec{E}$ and $\vec{v}$ along specified directions and the intensity with the field reversed, $I(-\overrightarrow{\mathrm{E}}, \overrightarrow{\mathrm{v}})$, or with $\overrightarrow{\mathrm{v}}$ reversed, $I(\overrightarrow{\mathrm{E}},-\overrightarrow{\mathrm{v}})$, is proportional to the $S P$-coherence term, or more generally to the even-odd coherence terms.

Since the initial state of the system composed of the internal variables of ion plus target is invariant under inversion, we have

$$
\sigma_{j i}(-\overrightarrow{\mathrm{v}})=P \sigma_{j i}(\overrightarrow{\mathrm{v}}) P=(-1)^{L_{i}-L_{j \sigma_{j i}}(\overrightarrow{\mathrm{v}})} .
$$

Similarly, the Hamiltonians relating to $\overrightarrow{\mathrm{E}}$ and $-\overrightarrow{\mathrm{E}}$ are related by $P$ according to

$$
P\left(H_{0}+V\right) P=H_{0}-V \text {. }
$$

Since $P^{2}=1$ and $P$ commutes with $S$, it follows that 


$$
\begin{aligned}
I(-\overrightarrow{\mathrm{E}}, \overrightarrow{\mathrm{v}}) & =C \sum_{i j}\left(i\left|P \exp \left(i H^{\dagger} t \mid \hbar\right) P S P \exp \left(-i H^{\dagger} t / \hbar\right) P\right| j\right) \sigma_{j i} \\
& =C \sum_{i j}(-1)^{L_{i}-L_{j}}\left(i\left|\exp \left(i H^{\dagger} t / \hbar\right) S \exp (-i H t / \hbar)\right| j\right) \sigma_{j i} \\
& =I(\overrightarrow{\mathrm{E}},-\overrightarrow{\mathrm{v}}) .
\end{aligned}
$$

In both cases we see from Eq. (5) that only the odd $\Delta L$ terms, i.e., the $S P$-coherence terms, contribute to the difference between the $\pm \overrightarrow{\mathrm{E}}$ or $\pm \overrightarrow{\mathrm{V}}$ intensities. This result is essentially similar to Eck's weak-field result. ${ }^{1}$ The only consequence of a nonperturbation analysis of $\mathrm{Ly}-\alpha$ is to multiply $\sigma_{S P \&}$ by a more complicated but fully calculable function of time.

We now consider measurements of light polarization as additional sources of information. This information will be represented in terms of two monitoring operators which are irreducible components of the tensor $\left.\vec{S}=\sum_{0} \vec{r}^{\prime} \mid 0\right)(0 \mid \vec{r}$. First we consider linear polarization or, more precisely, the difference of the intensity seen by a linear polarizer and one-third the average value of the intensity. This is proportional to

$$
S^{[2]}{ }_{0}=N\left(z z^{\prime}-r r^{\prime} / 3\right) P\left(\overrightarrow{\mathrm{r}}^{\prime}, \overrightarrow{\mathrm{r}}\right),
$$

where $\left.P_{f}\left(\vec{r}^{\prime}, \overrightarrow{\mathrm{r}}\right)=\sum_{0} \mid 0\right)(0 \mid, N$ is a normalization constant, and the $z$ axis is parallel to the axis of the polarizer. The total light emitted in one particular direction, the $z$ direction say, also measures the mean value of $S^{[2]}$ since

$$
\left[\left(x x^{\prime}-\frac{1}{3} r r^{\prime}\right)+\left(y y^{\prime}-\frac{1}{3} r r^{\prime}\right)\right] P_{f}=-\left(z z^{\prime}-r r^{\prime} / 3\right) P_{f} .
$$

Owing to the inversion symmetry of $S^{[2]}$, this operator will be represented by a straight line, not an arrow, in our pictorial models.

Second we consider circular polarization, which represents the difference between intensities with polarizations $\epsilon_{ \pm}=(x \pm i y) / \sqrt{2}$, i.e.,

$$
S^{[1]}{ }_{0}=\frac{1}{2} i\left(x y^{\prime}-y x^{\prime}\right) P_{f} \text {. }
$$

Within a particular level, the mean value of $S^{[2]}{ }_{0}$ is proportional to the alignment expressed as the mean value of $3 J_{z}^{2}-J^{2}$, and the mean value of $S^{[1]}{ }_{0}$ is proportional to the orientation expressed as the mean value of $J_{z}$. Since $J_{z}$ is the operator for an infinitesimal rotation, it will be represented by a sense of rotation around a straight line. Owing to their properties when one reverses $\vec{E}$ or $\vec{v}[$ (Eq. (5)], the beam and the field are represented by arrows for $S P$-coherent excitation and by straight lines for non-SP-coherent excitation.

We will discuss the inducing of alignment and orientation of the excited state or, equivalently, linear and circular polarization of the decay radiation, by exhibiting operator equations which relate the time variation of one operator to the instantaneous value of another whose initial mean value is nonzero. ${ }^{6}$ In the situation considered by Eck with $\overrightarrow{\mathrm{E}} \| \overrightarrow{\mathrm{v}}$ the $S P$ coherence has two effects: It alters both the total light intensity and its linear polarization. Here the appropriate equations of motion are, with $\vec{p}$ the electronic linear momentum in the center of mass,

$$
\begin{aligned}
& \frac{d}{d t} \dot{p}^{2}=-i\left[p^{2},-\overrightarrow{\mathrm{r}} \cdot \overrightarrow{\mathrm{E}}\right]=-2 \overrightarrow{\mathrm{p}} \cdot \overrightarrow{\mathrm{E}}, \\
& \frac{d}{d t}\left(p_{z}^{2}-\frac{1}{3} p^{2}\right)=2\left(p_{z} E_{z}-\frac{1}{3} \overrightarrow{\mathrm{p}} \cdot \overrightarrow{\mathrm{E}}\right),
\end{aligned}
$$

since by the Wigner-Eckart theorem the mean value of $p^{2}$ is proportional to the light intensity and the mean value of $p_{z}^{2}-\frac{1}{3} P^{2}$ is proportional to the alignment. Now the mean value of $p_{z}$ is proportional to the imaginary part of $\sigma_{S P z}$; thus the light emitted in any direction, i.e., the alignment, will alter as long as $E_{z} \neq 0$ and $\sigma_{S P_{z}} \neq 0$. When $E_{z}=0$, the mean value of the right-hand side of Eq. (6) vanishes, and altering the sign of $\vec{E}$ does not influence the intensity measured in any direction in first order, although it may be altered in higher orders, or by particular polarization components. We will now show explicitly that the light intensity emitted perpendicular to $\vec{v}$ is unaltered by changing the direction of $\vec{E}$, when $\overrightarrow{\mathrm{E}} \cdot \overrightarrow{\mathrm{v}}=0$. On the other hand, light polarized neither parallel nor perpendicular to $\vec{v}$ is sensitive to field direction even when $\overrightarrow{\mathrm{E}} \cdot \overrightarrow{\mathrm{v}}=0$.

We use reflection symmetry in the plane perpendicular to $\vec{v}$ to demonstrate the first point. The $p z$ wave function changes sign under this reflection, but the Hamiltonian is unaffected, as is the operator $-\left(z z^{\prime}-\frac{1}{3} r r^{\prime}\right) P_{f}$. It follows that the coefficient of $\sigma_{S P z}$ in Eq. (1) equals the negative of itself and must vanish, and then that any variation of intensity in this experiment is related to non-SP-coherent excitation.

Now consider the difference between two alignments at $\pm 45^{\circ}$ to the beam axis (Fig. 1). This difference relates to the mean value of the operator

$p_{x} p_{z}+p_{z} p_{x}=\frac{1}{2}\left\{\left[\frac{1}{2}\left(p_{x}+p_{z}\right)^{2}-\frac{1}{3} p^{2}\right]-\left[\frac{1}{2}\left(p_{x}-p_{z}\right)^{2}-\frac{1}{3} p^{2}\right]\right\}$,

and from the operator equation

$$
\frac{d}{d t}\left(p_{x} p_{z}+p_{z} p_{x}\right)=2 p_{z} E_{x}
$$




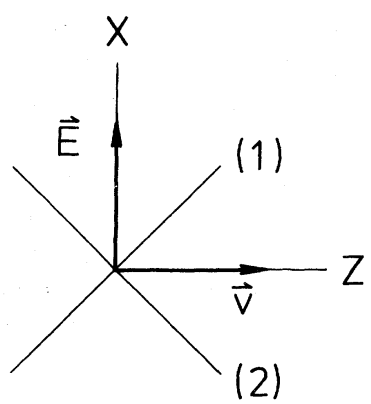

(a)

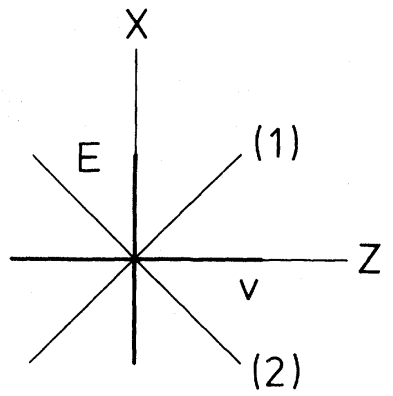

(b)

FIG. 1. Alignment at $\pm 45^{\circ}$. With $S P$ coherence, case (a), $+45^{\circ}$ (line 1 ) and $-45^{\circ}$ (line 2) are distinguishable, while without $S P$ coherence, case (b), the two lines are indistinguishable. Correspondingly, the two alignments are unequal if there is $S P$ coherence, but are equal without $S P$ coherence.

we see that this difference does not vanish if there is $S P$ coherence. In our representation of Fig. 1 detection arrangements (1) and (2) are clearly equivalent in case (b) - of non-SP-coherent excitation-but are inequivalent in case (a) - of $S P$-coherent excitation, so that we detect in this case only signals related to $S P$-coherent excitation.

Finally consider orientation (Fig. 2). The vector $\langle\vec{J}\rangle$ can have no component in the plane of $\vec{E}$ and $\vec{V}$ owing to reflection symmetry in this plane. When $v \perp E$, the plane perpendicular to $\overrightarrow{\mathrm{v}}$ is also a plane of symmetry for non-SP-coherent excitation. Accordingly the axial vector $\vec{J}$ can have no component in this plane. On the contrary with $S P$ coherence we have orientation according to

$$
\frac{d}{d t} \overrightarrow{\mathrm{J}}=-e \overrightarrow{\mathrm{r}} \times \overrightarrow{\mathrm{E}}
$$

where the mean value of $\vec{r}$ is proportional to the real part of $\sigma_{S P z}$. Note that now $\langle\overrightarrow{\mathrm{r}}\rangle \times \overrightarrow{\mathrm{E}}$ and hence $\langle\vec{J}\rangle$ are perpendicular to $\overrightarrow{\mathrm{v}}$. Correspondingly, in Fig. 2 in the case (a) of $S P$-coherent excitation, the two senses of rotation about $\vec{E} \times \vec{v}$ are distin-

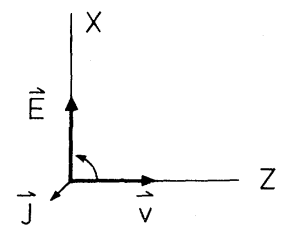

(a)

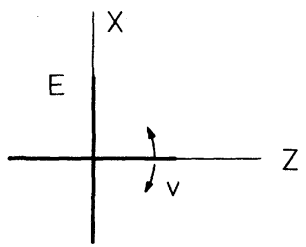

(b)

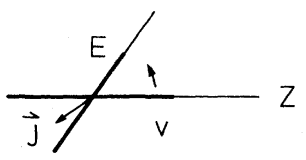

(c)

FIG. 2. Sense of rotation determined in case (a) with $S P$ coherence. Without $S P$ coherence, a sense of rotation is determined only if $E$ and $v$ are neither perpendicular (b) nor parallel. Figure 2(c) shows such a case. Here a sense of rotation is defined. Corresponding to this rotation, a nonzero mean orientation $\left\langle J_{y}\right\rangle$ is induced.

guishable, while in the case (b) of non-SP-coherent excitation they are not. The previously noted effect of orientation with non-SP-coherent excitation exists only when $\overrightarrow{\mathrm{E}}$ and $\overrightarrow{\mathrm{v}}$ are not perpendicular. Figure 2 (c) shows that in this case the two senses of rotation are indeed distinguishable.

It of ten proves convenient to apply a magnetic field $\vec{B}$ at right angles to $\vec{v}$ to induce a motional electric field $\overrightarrow{\mathrm{E}}=-(e / c) \overrightarrow{\mathrm{V}} \times \overrightarrow{\mathrm{B}}$ on the moving atoms. ${ }^{7}$ This must be avoided in studies of $S P$ coherence since it gives very confusing information. If one neglects spin effects, the only effect of the magnetic field is to rotate the wave function as a whole. In this case, with $\overrightarrow{\mathrm{B}}$ and $\overrightarrow{\mathrm{E}}$ perpendicular to $\vec{v}$, this rotation makes the collision-induced dipole moment nonperpendicular with respect to $\overrightarrow{\mathrm{E}}$. The mean value of $\overrightarrow{\mathrm{p}} \cdot \overrightarrow{\mathrm{E}}$ is nonzero, and the intensity will depend upon $\sigma_{S P z}$. This intensity is nevertheless unchanged when one reverses the direction of $\vec{B}$ (and therefore of $\vec{E}$ ), because the sense of rotation also changes. Moreover, if one takes into account spin-orbit interaction, there is an orientation signal in the $\vec{B}=\vec{V} \times \vec{E}$ direction due to spin uncoupling, ${ }^{8}$ even in the absence of an electric field and $S P$ coherence.

In summary, applying an electric field to excited 
hydrogenic atoms induces both alignment [Eq. (6)] and orientation [Eq. (8)] if there is $S P$ coherence. Such induction of orientation is favored when $\overrightarrow{\mathrm{E}}$ is perpendicular to $\vec{v}$, but alignment tends to be induced for all directions of $\overrightarrow{\mathrm{E}}$. Orientation by a field acting on aligned atoms occurs only when $\vec{E}$ and $\vec{v}$ are not perpendicular and can therefore be distinguished from alignment by a field acting on $S P$-coherently excited states.

Similarly alignment induced by $S P$-coherent ex- citation can be distinguished, when $\vec{v}$ is perpendicular to $\overrightarrow{\mathrm{E}}$, from that due to non-SP-coherent excitation because the alignments are not in the same direction.

We emphasize that all of our considerations are quite general and apply to any group of hydrogenic states with principal quantum number $n$.

One of us (J. H. M.) thanks the Freie Universität for support during the course of this work.
*Work supported in part by NSF under Grant No. GP39310.

$\dagger$ Permanent address: Behlen Laboratory of Physics, University of Nebraska, Lincoln, Neb. 68508.

${ }^{1}$ T. G. Eck, Phys. Rev. Lett. 31, 270 (1973).

${ }^{2}$ This experiment has now been carried out by two groups. See I. A. Sellin, J.`R. Mowat, R. S. Peterson, P. M. Griffin, R. Laubert, and H. H. Haselton, Phys. Rev. Lett. 31, 1335 (1973); A. Gaupp, J. Andrä, and J. Macek, Phys. Rev. Lett. 32,6 (1974).

${ }^{3}$ M. Lombardi and M. Giroud, C. R. Acad. Sci. (Paris)
266, 60 (1968).

${ }^{4}$ M. J. Alguard and C. W. Drake, Nucl. Instrum. Methods 110,311 (1970).

${ }^{5}$ U. F ano and J. Macek, Rev. Mod. Phys. 45, 553 (1973).

${ }^{6} \mathrm{M}$. Lombardi, Thesis (Grenoble, 1969) (unpublished); may be obtained from Microfilm No. A02894 CNRS 15 Quai Anatole France 75005, Paris, France.

${ }^{7}$ S. Bashkin, W. S. Bickel, D. Fink, and R. K. Wangsness, Phys. Rev. Lett. 15, 284 (1965).

${ }^{8}$ J. C. Lehmann, J. Phys. 25,809 (1964). 